\title{
Prevalence of BRCA1 Oncogen Expression in Breast Cancer Specimens of Patients with Positive Family History
}

\author{
Wijdan. H.Al- Dabbagh* \\ Nada.A.S.Al- Alwan** \\ Salim R. Al-Aubaidy***
}

\author{
MBChB \\ PhD (Path) \\ PhD (Path)
}

\begin{abstract}
:
Background: Breast cancer is the leading female cancer worldwide and in Iraq .Some mutations, particularly in BRCA1, significantly increase the risk of the disease.

Objectives: To demonstrate the frequency of BRCA1 in a group of high risk women with "positive family history" of breast cancer; correlating the immune expression of BRCA1 with some parameters of known prognostic significance.

Fac Med Baghdad 2015; Vol.57, No.3 Received: Feb, 2015 Accepted:June, 2015

Patients and Methods: Eighty-two female patients diagnosed with breast cancer (50 familial and 32 non familial) were included in the study. The mean age of the patients was 48.07. Immunohistochemistry was performed to assess the BRCA1 oncogene expression, Estrogen Receptor (ER), Progesterone Receptor (PR), Her 2 neu contents of the tumors.

Results: Out of the study group, $60.9 \%$ revealed family history of breast cancer. Forty-eight percent of the patients with positive family history were in the age group of 45 years or below; of those, $42.9 \%$ gave family history of cancer in their first degree relative while $25.7 \%$ had second degree relatives. BRCA1 positive expression was demonstrated in $39.02 \%$ out of all patients with breast carcinoma. In patients with positive family history for breast carcinoma, BRCA1 positive expression was demonstrated in $54 \%$ while in patients with negative family history it was $15.6 \%$. Among patients with positive BRCA1 expression $71.9 \%$ of those had negative ER expression and $57.9 \%$ exhibited negative PR expression.

Conclusions: BRCA1 gene expression determination in patients with positive family history should be promoted and validated to serve as indicators for early diagnosis of the disease.

Keywords: Breast, Cancer, BRCA1, ER, PR, Her2 neu, family history.
\end{abstract}

\section{Introduction:}

Breast cancer is the leading female cancer worldwide (1), and in Iraq (2). It has been recorded that in the UK and USA, approximately one in every ten women will contract the disease in their lifetime (3) . It is also amongst the leading cause of death in the female population in industrialized countries (1). Prognosis and survival rates for breast cancer vary greatly depending on the cancer type, stage, treatment, and geographical location of the patient. Survival rates in the Western world are high (4). It has been estimated that more than 8 out of 10 women (about 80\%) in England diagnosed with breast cancer survive for at least 5 years (5). In developing countries, however, survival rates are much poorer (5). Apart from positive familial history of breast cancer, the risk significantly increases in some mutations, particularly in BRCA1, BRCA2 and 553 genes expressions. In less than 5\% of cases, genetics plays a more significant role by causing a hereditary breast-ovarian cancer syndrome (6).This includes

*corresponding Auther: Oncology Teaching Hospital/Medical City. wijdan-hamza@yahoo.com

** Director, Iraqi National Cancer Research Center / Baghdad University.

***Dept. of Pathology, College of Medicine/ Baghdad University. those who carry the BRCA1 and BRCA2 gene mutations (7) . These mutations account for up to $90 \%$ of the total genetic influence with a risk of breast cancer of $60-80 \%$ in those affected (8) .The BRCA1 gene and its protein products have recently been the subject of intensive investigation because of their proven role in hereditary human breast and ovarian cancers (8) .

\section{Materials and Methods:}

This cross-sectional designed (retrospective) study and recruited a total of 82 female patients diagnosed with breast cancer at the Main Referral Training Center for Early Detection of Breast Tumors (Oncology Teaching Hospital/ Medical City) and the Iraqi National Cancer Research Center (Baghdad University).

The age of the patients in this study group ranged between 24 and 67 years. All patients were subjected to the Triple Assessment Technique (including Physical Breast Examination, Mammography and Fine Needle Aspiration Cytology FNA). Paraffin-embedded tissue blocks containing diagnostic histolological tissues of those patients were collected from February 2014. Nottingham modification of the Bloom- 
Richardson (9) was used in grading mammary carcinoma while staging was carried out according to the American Joint Committee on Cancer (AJCC)(10). Two histological types were identified: infiltrative ductal carcinoma) IDC73 - patients) and infiltrative lobular carcinoma (ILC - 9 patients).

Immunohistochemistry was performed utilizing four sections of 5 micron thickness, stained using Dako REAL ${ }^{\mathrm{TM}}$ EnVision $^{\mathrm{TM}}$ Detection System, Peroxidase / DAB. One section for assessing the BRCA1 oncogne using Anti-BRCA1, GLK-2,a mouse monoclonal antibody. A case of infiltrative ductal carcinoma of breast was used as a positive control. Two sections were stained for (ER) and (PR), using mouse IgG1 antibody to Estrogen byDako and monoclonal mouse IgG1 antibody to progesterone by Dako). Normal breast cells in the sample were used as internal positive controls for both ER and PR. The criteria of positive reaction for ER, PR included the observation of dark brown intra-nuclear precipitate. The forth section was treated by Her2neu monoclonal antibody (rabbit anti-human Her-2 protein byDako). Positive control was prepared from positive tumor control sections which were processed with each set of staining for the HER-2 immunohistochemistry. The membrane staining intensity and pattern of HER-2 / neu expression were considered for scoring according to the breast cancer HER-2/ neu scoring system (11).

In the current study, the positive BRCA1 reported only in the cytoplasm of tumor cells of breast cancer patients. The AntiBRCA1, GLK-2, a mouse monoclonal antibody used in this study, was directed against the C-terminal region of BRCA1 and seemed to reveal only the cytoplasmic form of this protein (12).

Tumor cells were scored depending on the percentage of labeled malignant cells: (13)

1. Score 0 (- ve) for BRCA1 : Tumor cells staining less than $10 \%$.
2. Score $1(+)$ : Staining observed in $10 \%-40 \%$.

3. Score $2(++)$ : Staining observed in $40 \%-70 \%$

4. Score $3(+++)$ : Staining observed in greater than $70 \%$.

Staining was considered positive when greater than $10 \%$ of the tumor cells showed positive staining (13).

Statistical Analysis: Statistical analysis was used to compare the clinicopathological data pertaining to breast cancer patients with positive family history of the disease with immunohistochemical expressions of BRCA1, ER, PR and Her2 neu.SPSS V. 17(statistical package for social sciences) was utilized for data input and analysis(14).

\section{Results:}

Eighty two female patients, who complained of breast lumps that were diagnosed as malignant, were enrolled in the study. Fifty of those patients had a positive family history (FH) of breast carcinoma (familial breast cancer) while 32 did not have any family history. The age range was (24-67 years) at the time of diagnosis with a mean of (48.07) and BMI of (30.38).

In those who had positive FH of breast cancer $(n=50), 28$ had an affected 1 st degree relative while 22 had an affected 2 nd degree relative.

The patients were divided into two groups according to BRCA1 oncogene expression : Those with -ve BRCA1 $(n=50)$ and those with +ve BRCA1 $(n=32)$; the latter was further sub divided into score $1(n=4)$, score $2(n=13)$ and score $3(n=15)$. In General, in our study all patients were divided to four main categories according to the FHx and BRCA1 immunohistochemistry results as shown in Figure (1).

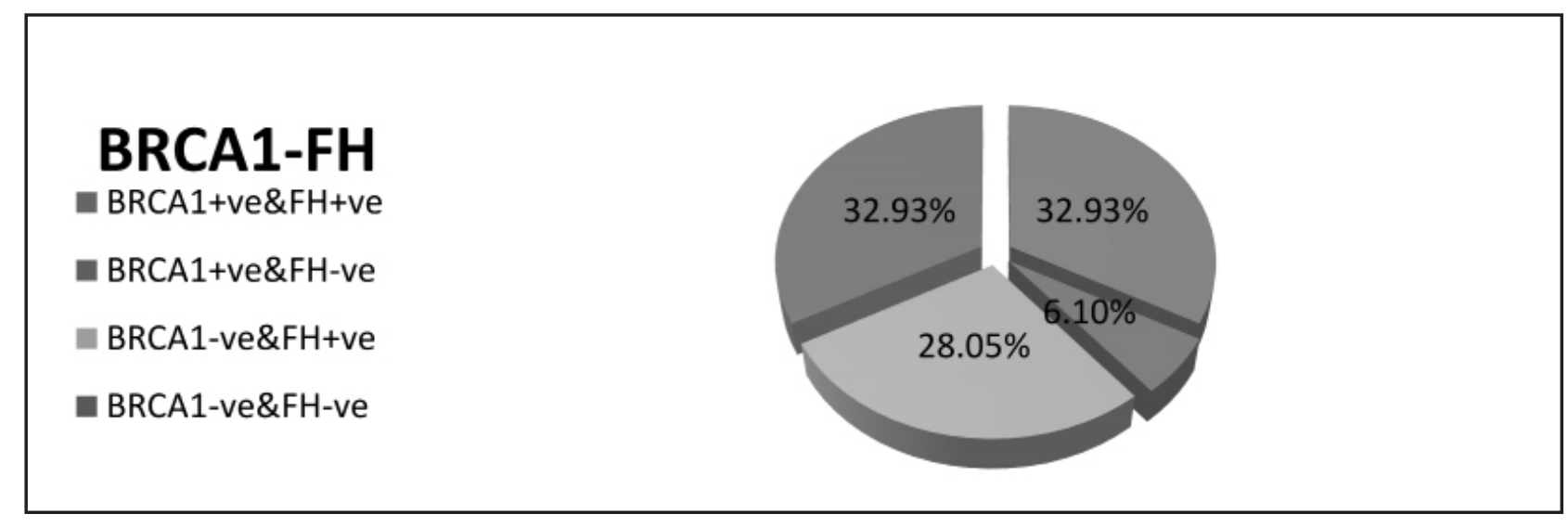

Figure (1): Pie Chart showing BRCA1 Oncogene Expression distribution in the study population sample in relation to the family history of breast cancer. 
Table (1): Distribution of BRCA1 Positive Oncogene Expression of the Study Population Sample in relation to the Family History of Breast Cancer.

\begin{tabular}{|c|c|c|c|c|}
\hline & & \multicolumn{2}{|c|}{ BRCA1 } & \multirow{2}{*}{ Total } \\
\hline & & BRCA1 -ve & BRCA1 +ve & \\
\hline \multirow{2}{*}{ No FH } & Count & 27 & 5 & 32 \\
\hline & \% within FH2x & $84.4 \%$ & $15.6 \%$ & $100.0 \%$ \\
\hline \multirow{2}{*}{ FH +ve } & Count & 23 & 27 & 50 \\
\hline & \% within FHx & $46.0 \%$ & $54.0 \%$ & $100.0 \%$ \\
\hline \multirow{2}{*}{ Total } & Count & 50 & 32 & 82 \\
\hline & \% within FHx & $61.0 \%$ & $39.0 \%$ & $100.0 \%$ \\
\hline
\end{tabular}

There was significant relationship between BRCA1 expression in those with family history of breast cancer P-value 0.001 ( $\mathrm{p}$-value $<0.05)$. The patients were divided into two age groups: first $(=<45$ year $)$ and second ( $>45$ year ).

Table (2) : Distribution of BRCA1 Expression and Family History of Breast cancer according to the Age group of the study population

\begin{tabular}{|c|c|c|c|c|c|c|c|}
\hline & & & \multicolumn{4}{|c|}{ BRCA1_FH } & \multirow[b]{2}{*}{ Total } \\
\hline & & & $\begin{array}{c}\text { BRCA+ve \& } \\
\text { FH+ve }\end{array}$ & $\begin{array}{c}\text { BRCA+ve \& } \\
\text { FH-ve }\end{array}$ & $\begin{array}{c}\text { BRCA-ve \& } \\
\text { FH+ve }\end{array}$ & $\begin{array}{c}\text { BRCA-ve \& } \\
\text { FH-ve }\end{array}$ & \\
\hline \multirow{4}{*}{ AgeGp } & \multirow{2}{*}{$=<45$} & Count & 19 & 3 & 5 & 8 & 35 \\
\hline & & \% within AgeGp & $54.3 \%$ & $8.6 \%$ & $14.3 \%$ & $22.9 \%$ & $100.0 \%$ \\
\hline & \multirow{2}{*}{$>45$} & Count & 8 & 2 & 18 & 19 & 47 \\
\hline & & \% within AgeGp & $17.0 \%$ & $4.3 \%$ & $38.3 \%$ & $40.4 \%$ & $100.0 \%$ \\
\hline \multirow{2}{*}{\multicolumn{2}{|c|}{ Total }} & Count & 27 & 5 & 23 & 27 & 82 \\
\hline & & \% within AgeGp & $32.9 \%$ & $6.1 \%$ & $28.0 \%$ & $32.9 \%$ & $100.0 \%$ \\
\hline
\end{tabular}

The peak expression was seen in the first age group with BRCA +ve \& FH+ve ( 54.3\%). There was significant statistical difference in the expression within the different two age groups P-value $0.002 \quad(<0.05)$.

Table (3) : Distribution of BRCA1 Expression and Family History of Breast cancer in relation to the tumor ER status.

\begin{tabular}{|c|c|c|c|c|c|c|c|}
\hline & & & \multicolumn{4}{|c|}{ BRCA1_FH } & \multirow[b]{2}{*}{ Total } \\
\hline & & & $\begin{array}{c}\text { BRCA+ve \& } \\
\text { FH+ve }\end{array}$ & $\begin{array}{c}\text { BRCA+ve \& } \\
\text { FH-ve }\end{array}$ & $\begin{array}{c}\text { BRCA-ve \& } \\
\text { FH+ve }\end{array}$ & $\begin{array}{c}\text { BRCA-ve \& } \\
\text { FH-ve }\end{array}$ & \\
\hline \multirow{4}{*}{ ER } & \multirow{2}{*}{-ve } & Count & 20 & 3 & 3 & 6 & 32 \\
\hline & & \% within ER & $62.5 \%$ & $9.4 \%$ & $9.4 \%$ & $18.8 \%$ & $100.0 \%$ \\
\hline & \multirow{2}{*}{$+v e$} & Count & 7 & 2 & 20 & 21 & 50 \\
\hline & & \% within ER & $14.0 \%$ & $4.0 \%$ & $40.0 \%$ & $42.0 \%$ & $100.0 \%$ \\
\hline \multirow{2}{*}{\multicolumn{2}{|c|}{ Total }} & Count & 27 & 5 & 23 & 27 & 82 \\
\hline & & \% within ER & $32.9 \%$ & $6.1 \%$ & $28.0 \%$ & $32.9 \%$ & $100.0 \%$ \\
\hline
\end{tabular}

In study sample ER -ve expression was seen in $(62.5 \%)$ of patients in group BRCA+ve \& $\mathrm{FH}+$. There was statistically significant association between the distribution of patients in the study group and the ER expression, P value 0.000 .

Table (4) : Distribution of BRCA1 Expression and Family History of Breast cancer according in relation to the tumor PR status

\begin{tabular}{|c|c|c|c|c|c|c|c|}
\hline & & & \multicolumn{4}{|c|}{ BRCA1_FH } & \multirow[b]{2}{*}{ Total } \\
\hline & & & $\begin{array}{c}\text { BRCA+ve \& } \\
\text { FH+ve }\end{array}$ & $\begin{array}{c}\text { BRCA+ve \& } \\
\text { FH-ve }\end{array}$ & $\begin{array}{c}\text { BRCA-ve \& } \\
\text { FH+ve }\end{array}$ & $\begin{array}{c}\text { BRCA-ve \& } \\
\text { FH-ve }\end{array}$ & \\
\hline \multirow{4}{*}{ PR } & \multirow{2}{*}{-ve } & Count & 18 & 4 & 6 & 10 & 38 \\
\hline & & \% within PR & $47.4 \%$ & $10.5 \%$ & $15.8 \%$ & $26.3 \%$ & $100.0 \%$ \\
\hline & \multirow{2}{*}{ +ve } & Count & 9 & 1 & 17 & 17 & 44 \\
\hline & & \% within PR & $20.5 \%$ & $2.3 \%$ & $38.6 \%$ & $38.6 \%$ & $100.0 \%$ \\
\hline \multirow{2}{*}{\multicolumn{2}{|c|}{ Total }} & Count & 27 & 5 & 23 & 27 & 82 \\
\hline & & \% within PR & $32.9 \%$ & $6.1 \%$ & $28.0 \%$ & $32.9 \%$ & $100.0 \%$ \\
\hline
\end{tabular}

PR -ve expression was observed in ( 47.4\%) of patients in the group displaying BRCA1+ve \& FH ve .

There was statistically significant association between the distribution of patients in the study group and the PR expression, $\mathrm{P}$ value $<0.009$. 
The frequency of diagnosing Infiltrative Ductal Carcinoma (IDC) was $89 \%$ among total patients in the study sample, while $10.9 \%$ were diagnosed with Infiltrative Lobular Carcinoma (ILC). Among those patients showing BRCA +ve \& FH +ve, IDC was seen in $81.4 \%$, and ILC in $18.5 \%$.

There was no statistically significant association between the distribution of patients in the study group and the following parameters: number of parity, contraceptive pill consumption, age at Menarche, Body Mass Index, tumor pathological type, stage of the disease, tumor grade and the lymph node status.

\section{Discussion:}

In Iraq, breast cancer constitutes about one fourth of the registered cancer cases among the Iraqi population according to the latest Iraqi Cancer Registry(2). World Health Organization (WHO) displayed that the low survival rates in less developed countries, including Iraq, is mainly attributed to the lack of early detection programs coupled with inadequate diagnostic and treatment facilities $(15,16)$.In the present series the average age of the patients at presentation was 48.07() years. Comparable findings were recorded in other Iraqi studies $(2,17$ ), which shows a trend for the disease to affect younger age groups. On the other hand, the peak age frequency of breast cancer in western countries is usually observed in the sixth decade and over(15,16 ).

In present study 50 patients $60.9 \%$ out of the total cases documented family history of breast cancer. It has been demonstrated that out of those patients aged $(<45$ years $)$, $42.9 \%$ gave family history of breast cancer in their first degree relative and $25.7 \%$ had second degree relatives. A Comparable study from India revealed $20.7 \%$ of patients with breast cancer had positive family history of cancer(18), while earlier studies on randomly selected breast cancer patients revealed that $14-16 \%$ had positive family history( 19 ). The higher rate of positive family history in our study is obviously attributable to the selection criteria(age and family history of breast cancer) in which 50 out of 82 patients documented a positive family history of breast cancer either in first or second degree relatives. Relative indications for testing for a mutation in BRCA1 include a family history among first or second, degree relatives with any of the following conditions: breast cancer diagnosed at age 50 or younger, primary multiple breast cancers in the same or opposite breast in an individual, ovarian cancer, both ovarian and breast cancers, breast cancer in males (20). Our aim was to target the cytoplasmic form of BRCA1 protein, considering that an abnormal location could be an indicator for BRCA1 mutation (at least in exon 11). Similar to other investigators, we attempted to chose a monoclonal antibody that has been shown to detect only the cytoplasmic form of BRCA1 protein (12). In our study the BRCA1 expression was significantly correlated with family history where by $54 \%$ of patients with positive family history had positive expression of BRCA1. In particular, BRCA1 positive expression was significantly correlated with the two stratified age groups «equal or less than 45 years» and «above 45 years». It was confirmed that that BRCA1 expression was statistically higher in the younger age groups (less than 45 years). Rather close findings were reported in another study from Egypt (21). and comparable results were also reported by a study from $\operatorname{Iraq}(22)$, while slightly higher frequencies were observed by others( 23) .Two studies from Kuwait(24) and Japan(25), showed positive BRCA1 staining in $64.6 \%$ and $65.7 \%$ of breast cancer specimens respectively.In a report from Algeria (26), it was observed that about $55 \%$ of breast cancer cases were diagnosed in women younger than 50 years. It was reported that the lower age of onset of breast cancer in Algerian could suggest the involvement of genetic factors such as mutation of BRCA1. In China (27), it was found that $71.42 \%$ of those with BRCA1 positive expression and positive family history belong to younger age groups. It has been proposed that these contradictory findings probably result from differences in the quality and specificity of the antibodies used, the different fixation methods utilized, quality of the archival paraffin embedded tissues which affect protein detection and the presence of splice variant isoforms of the BRCA1 protein in the tumors (28). In the present study among patients with BRCA1 positive expression, $71.9 \%$ revealed negative ER expression and $57.9 \%$ showed negative PR expression. It has been shown that most breast cancers in women with germ line BRCA1 mutations are estrogen receptor negative and typically lack expression of progesterone receptor $(\mathrm{PR})$ and human epidermal growth factor receptor (HER)2 over expression (the so-called 'Triple-Negative' breast cancers)(29,30) . It has been suggested that the association of ER negativity in breast cancer with BRCA1 mutation carrier status propose a tendency to regard all patients with ER positive tumors as non familial (31) .In China (27), it was revealed that $85.7 \%$ of patients with positive BRCA1 expression and family history of breast cancer express negative ER receptor content, while in Australia (32), 58.1\% of that group expressed negative ER receptor content. In USA(33), it was found that $77 \%$ of those with BRCA1 positive expression and positive family history had negative ER content and $86.7 \%$ showed negative PR. In the present study, there was no statistically significant correlation between BRCA1positive expression and Her2neu IHC expression. That might be attributable to the limited number of cases included and / or the quality of the utilized antibody survey.In this study, among patients with positive family history and BRCA1 positive expressions, $81.4 \%$ were diagnosed with IDC, while ILC was diagnosed in $18.5 \%$. It has been reported in the literature that patients with (IDC) have a poor survival compared to other types (34). Fisher 
(35). Other investigators demonstrated that most BRCA1associated ER negative tumors are often high-grade IDC (36). The present study found that $59.25 \%$ of breast carcinomas were graded as II while $40.7 \%$ were grade III . About $78 \%$ of cases with positive family history and BRCA1 positive expressions presented at the time of diagnosis with positive lymph node metastasis. Over $88 \%$ of cases with positive family history and BRCA1 positive expressions were diagnosed in Stages II, III and IV. That fact attracts the attention to the probability of high prevalence of poorly differentiated breast carcinomas among Iraqi patients that was emphasized earlier by the reports of Al Alwan from Iraq $(37,38)$; where she documented that $81 \%$ of her studied cases exhibited aneuploid DNA patterns with aggressive behavior $(39,19)$.

\section{Conclusions:}

BRCA1 expression had significant association with age, family history of the breast cancer and hormones (estrogen and progesterone) receptor contents. BRCA1 gene expression determination in patients with positive family history should be promoted and validated to serve as indicators for early diagnosis of the disease.

\section{Author Contributions:}

Nada.A.S.Al- Alwan: Direct supervisor on all work activities starting from preparation of specimens, clinical examination of cancer patients and follow up on center which she directing and writing.

Salim R.Al-Aubaidy : Supervisor, examination of the specimens and support in writing.

Wijdan.H.Al-Dabbagh: Selection of the study sample, acquisition of data and practical work in addition to writing.

\section{References}

1. International Agency for Research on Cancer (2010). Globocan 2008. World Cancer Statistics. Lyon, IARC Press.

2. Iraqi Cancer Registry 2010, Ministry of Health, Iraq, 2013(IVSL).

3.Robert E.Mansel, Oystein Fodstad and Wen G.Jiang . Metastasis of Breast Cancer 2007 Vol 2; pag 1-5

4. "World Cancer Report”. International Agency for Research on Cancer. 2008. Retrieved 2011-02-26.

5. Tsion Solomon, Bernard Rachet, Michal P Coleman et al Cancer Survival in England, patients diagnosed 2007-11, followed up to 2012.

6. Gage, M; Wattendorf, D; Henry et al "Translational advances regarding hereditary breast cancer syndromes (1 April 2012). “. Journal of surgical oncology 105 (5): 44451.

7.Boris Pasche, Steven T Rosen exal . Cancer Genetics( Cancer Treatment and Research). Berlin: 2010. pp. 19-20.
8. Scully R., Chen J., Ochs R.L et al . Dynamic changes of BRCA1 subnuclear location and phosphorylation state are initiated by DNA damage. Cel (1997) 90 ; 425-435.

9. National Cancer Control Programs. Policies and managerial guidelines, 2nd. ed. Geneva, World Health Organization, 2002.

10. Breast. In: Americo Joint Committee on Cancer: AJCC cancer staging Manual. 5th ed. Philadelphia, PA: Lippincott. Raven Publisher, 1997: 171-180.

11. Slamon DJ, Clark GM, Wong SG, et al. Human breast cancer: correlation of relapse and survival with amplification of the HER-2/neu oncogene. Science. 1987;235:177-82.

12. Kashima K, Oite T, Aoki Y, et al. Screening of BRCA1 mutation using immunohistochemical staining with C-terminal antibodies in familial ovarian cancers. Jpn J Cancer Res 2000; 91:399-409.

13. Yang O., Sakuria T.,et al.,: Prognostic significance of BRCA1 expression in Japanese sporadic breast carcinoma .American Cancer Society,2001; July 1,Volume 92/Number 1:54-60.

14.Chap TLE. Introductory biostatistics. By John Wiley \& Sons, Inc./New Jersey, 2003.

15. Siegel, R., Naishadham, D. \& Jemal, A. Cancer statistics,. CA Cancer J Clin 2013; 63, 11-30 (2013).

16 . Al Alwan $N$ \& Mualla F: Promoting Clinical Breast Examination as A screening Tool for Breast Cancer in Iraq Iraqi National Journal Of Nursing Specialties ,Vol 27 )1( ,2014.

17. Al-Alwan N, Al-Kubaisi W \& Al-Rawaq K: "Assessment of Response to Tamoxifen in Iraqi Patients with Advanced Breast Cancer". EMHJ, WHO. Eastern Mediterranean Regional Office, 2000; 6 (3/4): 475-482.

18. Janabi A.Al- A. immunohistochemical study of p53-oncosuppressor gene in correlation to other biochemical markers in breast cancer (a prospective study) Thesis. 2003.

19.Al-Alwan N.A.S.: Clinicopathological evaluation of nuclear D.N.A ploidy and hormone receptor contents in breast tumors. Ph.D. thesis submitted to the college of Medicine and the Committee of post graduate studies of the University of Baghdad. 1998.

20. Greenberg RA, "BRCA mutations and childhood cancer". Cancer Biol. Ther. (September 2006); 5 (9): 1103-4.

21. Manal Kamal, Khadiga ABU Gabal, Samia EL-Naggar, Yasser Hassan Shaaban, Mohamed Shehata, BRCA1 gene expression in breast cancer in relation to other prognostic markers in Egyptian women, AnationalJournal of obstetric and gynocolog ,ISSN:1308-8254, 2011.

22. Albederi F, Sabah A, Yassin A: Immunohistochemical study of breast carcinoma in old age Iraqi women by application of BRCA1 and P53. Kufa Med.Journal 2012.VOL.15.No.1.

23.Abdulla W.: A prospective study for the detection of 
Cytomegalovirus and BRCA1Gene in breast cancer using immunohistochemistry technique. A thesis submitted to the college council of health and medical technology as partial fulfillment of the requirement for the degree of master of technology in medical laboratory, supervised by Dr. M.A. Ali; 2010.

24. Al-Mulla F, Abdulrahman M,Varadharaj G, et al. BRCA1 gene expression in breast cancer: A correlative study between real time RT-PCR and immunohistochemistry. J Histochem Cytochem 2005; 53:621-9.

25.Yang O., Sakuria T.,et al.,: Prognostic significance of BRCA1 expression in Japanese sporadic breast carcinoma .American Cancer Society,2001; July 1,Volume 92/Number 1:54-60.

26. Uhrhammer N, Abdelouaha A, Lafarge L, Feillel V, Ben Dib A, Bignon Y. BRCA1 mutations in Algerian breast cancer patients: high frequency in young, sporadic cases. Int. J. Med. Sci. 2008, 5197.

27. A Pérez-Vallés, M Martorell-Cebollada, E NogueiraVázquez, et al the usefulness of antibodies to the BRCA1 protein in detecting the mutated BRCA1 gene. An immunohistochemical study, J Clin Pathol 2001;54:6 476-480.

28. Wilson CA, Ramos L, Villaseñor MR, et al. Localization of human BRCA1 and its loss in high-grade, non-inherited breast carcinomas. Nat Genet 1999;21:236-40.

29. .Lakhani SR, Vijver Van De MJ, Jacquemier J, Anderson TJ, Osin PP, McGuffog L, Easton DF: The pathology offamilial breast cancer: predictive value of immunohistochemical markers estrogen receptor, progesterone receptor, HER-2, and p53 in patients with mutations in BRCA1 and BRCA2. J Clin Oncol 2002, 20:2310-2318.

30. Atchley DP, Albarracin CT, Lopez A, et al: Clinical and pathologic characteristics of patients with BRCA-positive and BRCA-negative breast cancer. J Clin Oncol 2008, 26:42824288.

31. Tung N, Wang Y, Collins LC, et al . Estrogen receptor positive breast cancers in BRCA1 mutation carriers: clinical risk factors and pathologic features. BreastCancer Res 2010, 12:R12.

32. Sunil Badve, David J Dabbs, Stuart J Schnitt, et al . Basal-like and triple-negative breast cancers: a critical review with an emphasis on the implications for pathologists and oncologists Modern Pathology (2011) 24, 157-167

33.Tung, Nadine, Alexander Miron, Stuart J. Schnitt, et al.. Prevalence and predictors of loss of wild type BRCA1 in estrogen receptor positive and negative BRCA1-associated breast cancers. Breast Cancer Research 2010; 12(6): R95.

34. Greenberg RA, "BRCA mutations and childhood cancer". Cancer Biol. Ther. (September 2006); 5 (9): 1103-4 35. Fisher CJ, Egan MK, Smith P, Wicks K, Millis RR, Fentiman IS: Histopathology of breast cancer in relation to age. $\mathrm{Br} J \mathrm{Ca}$
1997; 75:593-6.

36. Armes JE, Egan AJ, Southey MC, Dite GS, McCredie MR, Giles $G G$, JL, Venter DJ: The histologic phenotypes of breast carcinoma occurring before age 40 years in women with and without BRCA1 or BRCA2 germline mutations: a populationbased study. Cancer 1998, 83:2335-2345.

37. Al-Alwan N. DNA proliferative index as a marker in Iraqi aneuploid mammary carcinoma. Eastern Mediterranean Health Journal, 2000, 6 (5/6):1062-1072.

38.Data were provided by the Northern Ireland Cancer Registry on request, June 2013.

39.Olson, James Stuart. Bathsheba's Breast: Women, Cancer and History. Baltimore: The Johns Hopkins University (2002); Press. pp. 199/200. ISBN 0-8018-6936-6. 